\title{
TEORÍA DE LA SUBJETIVIDAD Y PSICOTERAPIA: UNA PROPUESTA DESDE LA PERSPECTIVA HISTÓRICO CULTURAL do
}

\author{
Jeremy Swan Rodriguez-Camejo ${ }^{1}{ }^{\circledR}$, Tania García-Ramos $\mathbb{D}^{\mathbb{D}}, \&$ Sara \\ Santiago-Estrada ${ }^{(1)}{ }^{2}$
}

Universidad de Puerto Rico, San Juan, Puerto Rico ${ }^{3}$.

\begin{abstract}
RESUMEN
En este artículo se discute el valor de la teoría de la subjetividad, desde la Perspectiva Histórico Cultural (PHC), para el campo de la psicología clínica y la psicoterapia. Para esta discusión se explora cómo la traducción y difusión de la obra de Vygotsky en Occidente se vio afectada por diversos factores históricos e ideológicos que impidieron que la PHC se extendiera al campo de la psicología clínica. Además, se discute la propuesta teórica de la subjetividad, la psicoterapia y la Epistemología Cualitativa que elaboró González Rey como una alternativa para generar un puente entre la PHC y la psicología clínica. Al final se discuten aspectos que deben elaborarse en la propuesta de González Rey en su implementación en el campo clínico.
\end{abstract}

Palabras Claves

subjetividad; perspectiva histórico cultural; psicología clínica; psicoterapia; epistemología cualitativa

\begin{abstract}
This article discusses the value of the theory of subjectivity of the Cultural Historical Perspective (CHP) for the field of clinical psychology and psychotherapy. In this discussion we explore how the translation and diffusion of Vygotsky's work in the west was affected by historical and ideological factors that prevented the CHP from extending its reach to the field of clinical psychology. Additionally, we discuss the theory of subjectivity, psychotherapy and qualitative epistemology proposed by Gonzalez Rey as an alternative that could be the link between the CHP and clinical psychology. Finally, we discuss aspects that need to be further elaborated in Gonzalez Rey's theory as part of its implementation in the clinical field.
\end{abstract}

Keywords

subjectivity; cultural historical perspective; clinical psychology; psychotherapy; qualitative epistemology

\footnotetext{
1 Correspondence about this article should be addressed to Jeremy Swan Rodriguez-Camejo: jsrodriguezcamejo@gmail.com

2 Conflicts of Interest: The authors declare that the research was conducted in the absence of any commercial or financial relationships that could be construed as a potential conflict of interest.

3

Recinto Río Piedras.
} 


\section{THEORY OF SUBJECTIVITY AND PSYCHOTHERAPY: A PROPOSAL FROM THE HISTORICAL-CULTURAL PERSPECTIVE}

\section{Introducción}

En décadas recientes, varios autores de la psicología se han interesado por la obra de Vygotsky y la Perspectiva Histórico Cultural (PHC) en general. Esto ha permitido que este acercamiento se desarrolle en el contexto educativo (González Rey, Mitjans Martínez \& Bezerra, 2016; Rodríguez Arocho 1995, 1999, 2006). Sin embargo, la PHC no ha incidido de forma significativa en la psicología clínica. Esta situación parece estar cambiando pues, en tiempos recientes, se han discutido las posibles aplicaciones de la PHC al campo de la clínica, en particular a la psicoterapia (Alonso, 1998; De Oliveira, Goulart \& González Rey, 2017; Díaz-Gómez, González Rey \& Arias-Cardona, 2017; Echemendía-Tocabens, 2013; González Rey, 2009c, 2011b, 2015; González Rey, Goulart \& Bezerra, 2016; Hernández, 2010; Holzman, 2014; Kats \& Hadas, 1995; Kozulin, 1990; Miltenburg \& Singer, 1999; Portes, 2011; Ryle, 1991; Wilson \& Weinstein, 1992; Zaldívar Pérez, 1999).

El propósito central de este ensayo es discutir el valor de la teoría de la subjetividad, desde la Perspectiva Histórico Cultural (PHC), para el campo de la psicología clínica y la psicoterapia. Para realizar lo anterior, discutiremos la teoría de la subjetividad que desarrolló González Rey (2002), y otras aportaciones de este y otros autores (De Oliveira et al., 2017; Díaz-Gómez et al., 2017; González Rey, 2009c, 2011b, 2015; González Rey et al., 2016). Comenzaremos este recorrido exponiendo el contexto histórico en el cual toma lugar la traducción y difusión de la obra de Vygotsky en Occidente. Continuaremos identificando posibles limitaciones en la aplicación de la PHC al contexto clínico. Luego presentaremos la propuesta de González Rey a través de tres ejes temáticos: (1) su propuesta teórica, (2) su propuesta para la psicoterapia, y (3) su propuesta investigativa mediante una Epistemología Cualitativa. Al final, exponemos un análisis crítico en torno a asuntos a elaborar en la teoría de la subjetividad y su aplicación a la psicología clínica.

\section{Traducción y difusión de la obra de Vygotsky en Occidente}

La traducción y difusión de la obra de Vygotsky en Occidente transcurre en el contexto de la llamada revolución cognitiva que comenzó en la década de 1950 (Gardner, 1988; Miller, 2003; Rodríguez Arocho, 2010, 2013; Varela, 1988). Durante el desarrollo 
inicial de la revolución cognitiva, la perspectiva de procesamiento de información, junto con la noción de representación, se tornó en el núcleo paradigmático más representativo de la psicología cognoscitiva (Rivière, 1987; Rodríguez Arocho, 1994, 2010).

Rodríguez Arocho (1995) indica que, al adoptar el procesamiento de información como su modelo más representativo, la psicología cognoscitiva se distanciaba de la explicación de los problemas que le habían dado origen. Es entonces que, en el marco de estas problematizaciones, se comienza a descubrir en Occidente el legado de otros autores que ofrecían explicaciones alternas en torno al tema de la cognición. Es en este contexto que se desarrollan las aportaciones de Lev S. Vygotsky en Occidente (Rodríguez Arocho, 1994).

Para comprender las aportaciones de Vygotsky en Occidente es importante considerar los periodos de su obra. Según González Rey (2009b, 2009c, 2016), la obra de Vygotsky se puede dividir en tres periodos que totalizan aproximadamente 15 años. El primer periodo tomó lugar desde 1919 a 1927 durante el cual el psicólogo ruso mantuvo una preocupación explícita por los temas de la personalidad y de la motivación humana. Mediante estos se expresa su interés por los aspectos subjetivos de la psique (González Rey, 2007b, 2008, 2009a, 2010a, 2013a, 2014b, 2016). El segundo periodo de la obra de Vygotsky se extendió desde 1928 a 1931. En ese segundo momento de su obra, Vygotsky se centró en las categorías de: función psíquica superior, signo, mediación semiótica e interiorización. El tercer y último periodo de Vygotsky, tomó lugar desde el 1932-1934 (González Rey, 2007b, 2008, 2009a, 2010a, 2013a, 2016). En este, Vygotsky revisita algunos de los conceptos del primer periodo de su obra, brindándole importancia a las emociones, la imaginación, la personalidad, entre otros conceptos que acentuaban la relevancia de lo afectivo en la psique. Entre estos conceptos se encontraban: el sentido, la situación social del desarrollo y la vivencia (perezhivanie) (González Rey, 2014b).

Durante la revolución cognitiva, gran parte de los lectores de la obra de Vygotsky se concentraron en la producción teórica del segundo periodo de su vida. Esta es la interpretación dominante sobre este autor en Occidente y en la psicología soviética (González Rey, 2009b, 2016; Rodríguez Arocho, 2010). Algunos autores identifican este periodo como el surgimiento de la PHC (Van der Veer y Valsiner, 1991). De esta forma, el hecho de que la obra de Vygotsky comenzara a leerse e interpretarse en Occidente en el contexto de la revolución cognitiva tuvo una influencia significativa en lo que conocemos de este autor. Este enfoque en la obra de Vygotsky, en el periodo en el cual se centró en la función psíquica superior y la mediación semiótica, dio paso al "sesgo 
cognitivista" (Rodríguez Arocho, 2013) o el "giro objetivista" (González Rey, 2009b) de la obra de Vygotsky.

El sesgo cognitivista se caracterizó por una lectura altamente selectiva y parcial de la obra de Vygotsky (Daniels, 2001; Rodríguez Arocho, 2013). Se enfocó primordialmente en conceptos vinculados a lo cognitivo y excluyó conceptos vinculados a la personalidad y las emociones, como fueron, por ejemplo, vivencia (perezhivanie), situación social del desarrollo y sentido (González Rey, 2009a, 2014b; Rodríguez Arocho, 2013). Lo anterior desembocó en una simplificación de las ideas expresadas por Vygotsky (Ageyev, 2003; González Rey, 2009a; Rodríguez Arocho, 2013).

\section{Perspectiva histórica cultural y la psicología clínica}

En la psicología clínica, y en otras especialidades de la salud mental, la PHC ha recibido poca atención, en comparación con otros campos (Portes, 2011). En disciplinas como la psicología del desarrollo, psicología educativa y educación, la influencia de la PHC ha sido extensa (Daniels, 2001; González Rey et al., 2016; Rodríguez Arocho, 1995, 1999, 2006). El sesgo cognitivista ha jugado un papel importante en limitar que la PHC se extienda al campo de la psicología clínica, ya que se excluyeron aspectos de la obra de Vygotsky que son imprescindibles para la elaboración de una propuesta para la psicoterapia. Se excluyeron: la importancia a las emociones, el carácter generador de la psique, la unidad de lo cognitivo y lo afectivo, y el desarrollo de una representación de la mente apoyada en una perspectiva cultural-histórica (González Rey, 2007b, 2008, 2009a, 2010a, 2013a, 2016).

Sin embargo, el que la PHC no haya incursionado de forma profunda el campo de la psicología clínica no se limita al sesgo cognitivista. Existen otros factores que contribuyeron a esto. En primer lugar, la salud mental, la consejería y la psicoterapia no eran asuntos que interesaran de forma directa a Vygotsky, Luria y la PHC en general (Portes, 2011). Su interés era desarrollar una psicología general para la nueva y emergente psicología soviética de corte Marxista. En segundo lugar, la consejería y la psicoterapia, como prácticas culturales, estaban comenzando a surgir de prácticas basadas en modelos psicodinámicos y conductistas en Occidente durante la primera mitad del siglo XX (Portes, 2011).

En tercer lugar, Vygotsky no logró desarrollar de forma profunda ciertos aspectos de su teoría (González Rey, 2016). Específicamente, no pudo elaborar aspectos como la 
personalidad y las emociones, con la profundidad que presentó el desarrollo, el aprendizaje y las funciones psíquicas superiores.

El cuarto y último factor es que, por largas décadas, en la psicología rusa no hubo desarrollos significativos de la psicología clínica y la psicopatología (González Rey, 2009c). Esto en sí mismo se debe a limitaciones de orden político, institucional y teóricoprofesional, que dificultaron el desarrollo de la psicología clínica dentro del marco histórico cultural que caracterizó el desarrollo de la psicología soviética (González Rey, 2008, 2009c, 2013c, 2014a).

Las limitaciones de orden político estaban asociadas a diferentes asuntos; (1) la exclusión del psicoanálisis, considerado como "ciencia burguesa", lo que colocó en entredicho sus prácticas (González Rey, 2009c); (2) la explicación de la sociedad y el ser humano desde el marxismo dejaba poco margen para temas como la subjetividad o los 'trastornos'; (3) según la visión oficial, en el ser humano predominaban la conciencia y la voluntad, convertidos en valores sociales de significación política (González Rey, 2009c). Esto generó el voluntarismo, que privilegiaba la capacidad absoluta de la voluntad para superar los problemas. La combinación de estos elementos, valores ideológicos de la dirección política, dificultó el ejercicio de la clínica (González Rey, 2009c).

En el orden institucional, la psicología soviética fue hegemonizada por la visión objetivizante y positivista de la Teoría de la Actividad (González Rey, 2009c, 2013c, 2014a, 2014b). Esta teoría, una vez se instituyó, privilegió la formación de cuadros y delimitó los espacios institucionales y publicaciones congruentes con esa perspectiva. Finalmente, en el orden teórico-profesional se encontraba la división rígida entre las ciencias, según la cual los llamados 'trastornos' y la 'enfermedad' se definirían desde el campo de la medicina. La psiquiatría no brindó espacio de desarrollo a la psicología en el área de la salud (González Rey, 2009c).

\section{Propuesta teórica: Una aproximación a la subjetividad desde la PHC}

Como señala Portes (2011), en la PHC no existió una intención dirigida al desarrollo de la clínica. Más bien, el enfoque estuvo dirigido a desarrollar una nueva concepción sobre la psique humana como parte de una Psicología General (González Rey, 2009c). Por esta razón, no debe pensarse que en la obra de Vygotsky se encuentran las categorías específicas para definir una opción histórica cultural en psicoterapia. Esto implica que hay un reto grande en la intención de extender la PHC al campo de la clínica. 
Este reto consiste en desarrollar la teoría más allá de las elaboraciones de Vygotsky, para lograr su aplicación al campo de la psicología clínica y de la psicoterapia. Esto es especialmente necesario cuando consideramos que las "semillas" iniciales para este esfuerzo pueden surgir del primer y tercer periodo de la obra de Vygotsky. En ambos se destaca la importancia de las emociones y la representación sistémica de la psique.

Las dificultades discutidas no significan, sin embargo, que no ha habido esfuerzos para aplicar la PHC a la psicología clínica. A lo largo de los años, han surgido algunas publicaciones en la que autores han aplicado conceptos centrales de este acercamiento a dicho campo (Alonso, 1998; Echemendía-Tocabens, 2013; González Rey, 2009c, 2011b; Hernández, 2010; Holzman, 2014; Kats \& Hadas, 1995; Kozulin, 1990; Miltenburg \& Singer, 1999; Portes, 2011; Ryle, 1991; Wilson \& Weinstein, 1992; Zaldívar Pérez, 1999). Ejemplos de esto lo son la zona de desarrollo próximo (Alonso, 1998), vivencia (Hernández, 2010) y las herramientas psicológicas (Miltenburg \& Singer, 1999). No obstante, la mayoría de estos esfuerzos no han sido sistemáticos. Estos autores han extrapolado conceptos de la PHC a la psicología clínica y/o a la psicoterapia sin una reconceptualización que permita el desarrollo de estas ideas dentro de dichos campos.

Por esta razón, nos estaremos centrando en las aportaciones de González Rey, según las elaboró en sus múltiples escritos y colaboraciones con otros autores, cuyas propuestas han sido las más desarrolladas en términos de la aplicabilidad de la PHC a la psicoterapia (De Oliveira et al., 2017; Díaz-Gómez et al., 2017; González Rey, 2009c, 2011b, 2015; González Rey et al., 2016). Discutiremos primero los planteamientos generales de su teoría para luego discutir la propuesta de la teoría de la subjetividad al ámbito clínico.

La aplicación de la PHC a la psicoterapia se posibilita por la teoría de la subjetividad y la Epistemología Cualitativa, que elaboró el psicólogo cubano Fernando González Rey (González Rey, 2002, 2007a). González Rey (2002) elaboró su teoría a partir de la obra de múltiples autores, entre los cuales se destaca a Vygotsky. Específicamente, se enfocó en su primer y tercer periodo, dirigidos a explicar la afectividad y el funcionamiento complejo de la psique. Las categorías de sentido (Vygotsky, 1982/1993), la importancia de la unidad afectivo-cognitiva (Vygotsky, 1982/1993), y de la unidad indisoluble del sujeto con su entorno (en el concepto de vivencia) (Vygotsky, 1934), sirvieron de base para las elaboraciones conceptuales de González Rey (2002). 


\section{La subjetividad}

La teoría de la subjetividad da cuenta de la subjetividad como objeto de estudio que tiene una naturaleza ontológica propia y que existe como realidad cualitativa distinguible de otras formas (González Rey, 2002). La subjetividad se define como "la producción simbólico-emocional que emerge ante una experiencia vivida, la cual integra lo histórico y lo contextual en el proceso de su configuración” (González Rey, 2011a, p.313). Desde esta perspectiva, la subjetividad es conceptualizada como un sistema que se articula de manera recursiva de dos momentos: la subjetividad individual y la subjetividad social. Esta se organiza en las prácticas de los individuos y grupos, expresando continuamente una tensión entre su organización inicial y las formas que toma en el curso de esas prácticas (González Rey, 2010b). Desde esta perspectiva, la subjetividad no se reduce a un estado interno. Por el contrario, es una dimensión compleja, que involucra tanto lo psicológico como lo social en una relación recursiva, y cuya naturaleza es histórica y social (Hernández, 2008).

\section{Sentidos subjetivos y configuraciones subjetivas}

Según González Rey (2002; 2011a), los sentidos subjetivos son la unidad básica y constituyente de la subjetividad. Los define como (2001a):

Aquella unidad de los procesos simbólicos y emocionales donde la emergencia de uno de ellos evoca al otro sin convertirse en su causa, formando verdaderas cadenas con formas muy diversas de expresión según el contexto en que la persona está implicada (p.312).

El efecto de toda experiencia vivida entra en la subjetividad a través de los sentidos subjetivos, los cuales la persona produce a partir de su configuración subjetiva en ese momento particular. Los sentidos subjetivos son el proceso a través del cual toda experiencia es reconfigurada y se transforma en subjetiva.

González Rey (2002) afirmó que los distintos sentidos subjetivos que surgen en las acciones del individuo en el contexto cultural se organizan en sistemas psicológicos relativamente estables, conocidos como configuraciones subjetivas, que implican "la integración de elementos de sentido y significación que caracterizan la organización subjetiva de un ámbito de la experiencia del sujeto, y que asumen estructuras diferentes en el curso de sus acciones" (p.113). La configuración subjetiva, entonces, define las formas concretas de experiencia del sujeto en los espacios simbólicos de la cultura y es 
en estas configuraciones que surge toda función psicológica (Hernández, 2008), organizadas a partir de la historia individual del sujeto. La historia aquí no se limita a lo discursivo o lo narrativo, no se agota allí, sino que hace referencia al conjunto de experiencias emocionales que incide en que cada persona presente un carácter único e irrepetible (González Rey, 2011a).

Desde esta perspectiva, los procesos psicológicos mencionados no ocurren en el vacío, descontextualizados, ni desvinculados de otros individuos (González Rey, 2011a). El sujeto aquí ocupa un lugar central y se refiere a la persona singular, quien está configurada procesal, histórica y socialmente (Díaz-Gómez \& González Rey, 2005). El sujeto es definido como "aquel individuo o grupo, capaz de abrir nuevos caminos de subjetivación dentro del espacio normativo de su acción” (González Rey \& Mitjans Martínez, 2017, p.12). Junto al concepto de sujeto, González Rey y Mitjans Martínez (2017) integran el concepto de agente que se emplea para hacer referencia a las posiciones activas que un individuo o grupo asume en una actividad en curso, "pero que no genera nuevos caminos de subjetivación más allá de ellas” (p.12) Desde esta mirada, la persona no está determinada de manera alguna por la estructura o práctica social. El sujeto tiene una capacidad generadora diferenciada frente a lo vivido, que le permite múltiples opciones, decisiones y acciones, cargadas de sentidos subjetivos en el proceso de la experiencia. La persona se constituye en sujeto cuando es capaz de producir opciones que entran en tensión con los sistemas normativos hegemónicos del espacio social donde se lleva a cabo su acción, generando alternativas de sentidos subjetivos frente a dichos sistemas (González Rey, 2002).

\section{Desarrollo subjetivo}

González Rey y Mitjans Martínez (2017) distinguen entre el desarrollo psíquico y el desarrollo subjetivo. Ellos afirman que el desarrollo psíquico es anterior al subjetivo y se caracteriza por diversas conductas y operaciones en los primeros años de vida del niño (ej. La coordinación visomotora). De igual forma plantean que la psique no es exclusiva de la condición humana. Los procesos psíquicos más complejos, que son propiamente humanos, surgen en el proceso de un niño pasar de la psique a la subjetividad. Es decir, emergen cuando "su funcionamiento es regulado por configuraciones subjetivas que expresan la historia y el contexto actual del individuo" (González Rey \& Mitjans Martínez, 2017, p.6). Desde la niñez una persona comienza a producir sentidos subjetivos. Esto ocurre cuando las emociones del niño se comienzan a organizar de forma 
diferenciada en relación con quienes le rodean, muy especialmente, en la comunicación con los adultos.

El desarrollo subjetivo se genera en momentos en donde una persona enfrenta nuevas experiencias de vida y se ven en la necesidad de desarrollar nuevos recursos para vivirlas. Enfrentar nuevas experiencias y no poder desarrollar los recursos subjetivos necesarios para enfrentarlos implicará entrar en procesos de sufrimiento que lo paralizarán en su capacidad de producción subjetivas (González Rey \& Mitjans Martínez, 2017).

El surgimiento del desarrollo subjetivo siempre está vinculado a la generación de configuraciones subjetivas que implican diversas áreas de la vida del individuo y distintos recursos subjetivos. De esta forma, la producción de nuevos sentidos subjetivos y la formación de nuevas configuraciones subjetivas que permitan al individuo enfrentar nuevas experiencias de vida, son el motor del desarrollo subjetivo (González Rey \& Mitjans Martínez, 2017).

Los procesos de desarrollo subjetivo ocurren de forma única y diferenciada en cada persona. Las configuraciones subjetivas que permiten el desarrollo subjetivo pueden surgir en momentos diversos de la vida y ante experiencias completamente diferentes del transcurso existencial de un individuo. De igual forma los recursos subjetivos que propician el desarrollo subjetivo en un momento podrían ser un obstáculo para el desarrollo en otra instancia de la vida del individuo (González Rey \& Mitjans Martínez, 2017).

\section{Subjetividad social}

Un último concepto que es parte fundamental de la teoría de la subjetividad es la subjetividad social. González Rey (2008) la definió como,

...la forma en que se integran sentidos subjetivos y configuraciones subjetivas de diferentes espacios sociales, formando un verdadero sistema en el cual lo que ocurre en cada espacio social concreto como la familia, escuela, grupo informal, etc. está alimentado por producciones subjetivas de otros espacios sociales (p.234).

En su expresión, estas configuraciones se articulan estrechamente entre sí y establecen configuraciones subjetivas complejas en la organización social (González Rey, 2002). Según se plantea en este marco conceptual, la subjetividad social no es una entidad portadora de características universales estáticas. Por el contrario, esta es un sistema de 
organización cuyas características devienen de los espacios históricos socioculturales específicos dentro de los cuales se articula (González Rey, 2002).

Desde la subjetividad social, los procesos sociales coexisten con los individuales (González Rey, 2002). Los individuos son constituyentes y constituidos, de forma simultánea, en la subjetividad social. Sin embargo, añade el autor, la constitución del individuo en la subjetividad social no es un proceso que sigue rutas universales, ni definidas de forma estática por las características de los espacios sociales en los cuales estos viven. Por el contrario, es un proceso diferenciado en el que las consecuencias para las instancias sociales y para los individuos que las forman, dependen de los modos que adopten las relaciones entre lo individual y lo social. Ambos aspectos tienen un carácter activo, es decir, cada uno se configura de formas muy diversas ante la acción del otro, proceso que acompañará a ambos, el desarrollo social y el desarrollo individual (González Rey, 2002).

\section{Propuesta para la psicoterapia: La teoría de la subjetividad como alternativa para extender la PHC a la psicología clínica}

Una vez explicados los planteamientos principales de la teoría de la subjetividad, consideramos el valor de este marco conceptual como acercamiento para la clínica. Aclaramos que, aunque González Rey (2009c, 2011, 2015) aplicó su teoría de la subjetividad al contexto clínico, él no estaba de acuerdo con la idea de desarrollar una escuela de terapia desde la PHC. Según este autor, desarrollar una escuela de psicoterapia fundamentada en la perspectiva histórico cultural, reproduciría el dogmatismo teórico hermético de algunos acercamientos psicoterapéuticos. Según él, esto tiende a excluir el diálogo con otras posiciones y cierra la puerta a nuevos caminos en el desarrollo del conocimiento actual partiendo de la investigación y el cuestionamiento. Este autor defendió el carácter constructivo interpretativo de su propuesta histórica cultural, según la cual, la práctica está en continuo desarrollo para responder a las necesidades teóricas y prácticas que surjan (Díaz-Gómez et al., 2017). Los principios teóricos iniciales no son estáticos o incambiables, sino que están en movimiento a partir de las nuevas adquisiciones del conocimiento, en las diferentes áreas de investigación orientadas por este enfoque (González Rey, 2009c).

Desde este acercamiento, la psicoterapia es concebida como una práctica profesional de carácter relacional, dialógico, orientada a la producción de espacios alternativos para el desarrollo subjetivo en el momento actual de la vida de una persona 
(González Rey, 2009c; González Rey et al., 2016). El paciente es conceptualizado como un individuo o grupo social en diálogo con la posibilidad de emerger como agente de su proceso de vida cargado de emocionalidad, expresada mediante su subjetividad. El objetivo de la psicoterapia, desde esta mirada, es crear un espacio dialógico que genere apertura en el paciente a nuevos contextos de vida; donde este pueda emerger como sujeto de su proceso de vida y abrirse a nuevos caminos de subjetivación (González Rey \& Mitjans Martínez, 2017).

El terapeuta tendrá una posición simétrica con el paciente, en donde este último tendrá la posibilidad de preguntar y de asumir un lugar interpretativo. Es indispensable que el terapeuta y el paciente generen un diálogo espontáneo, reflexivo, activo y auténtico, en el cual ambos puedan asumir un lugar como sujetos (González Rey, 2009c). Se le brinda importancia central a la capacidad de los individuos, ciudadanos y grupos de posicionarse de manera activa en su vida, surgiendo como sujetos de sus propias prácticas (González Rey, 2009c; González Rey et al., 2016). En esta perspectiva, el profesional debe aceptar el reto de comunicarse con el otro a partir del lugar que ese otro asume en cada momento de la acción profesional (González Rey et al., 2016).

El terapeuta juega un papel activo en este acercamiento, busca construir y comprender las configuraciones subjetivas de la persona que atraviesa el problema, apoyándose en hipótesis que va elaborando a lo largo del proceso terapéutico (González Rey, 2009c). El saber del terapeuta se constituye en una herramienta facilitadora de hipótesis sobre la configuración subjetiva asociada en la emergencia de los síntomas. Esto permitirá pensar acciones y posiciones que impliquen a la persona en nuevas producciones subjetivas, que estimulen contradicciones que faciliten la emergencia de nuevos sentidos subjetivos. De esta forma se podrán iniciar caminos que integren la reflexión, las emociones, y los comportamientos en diferentes momentos asociados a nuevos espacios vitales y relaciones (González Rey, 2009c, 2011).

El terapeuta podrá participar e inducir tópicos de conversaciones en torno a áreas que puedan ser significativas por los sentidos subjetivos asociados a estas para el individuo o el grupo en terapia. El clínico, para levantar sus hipótesis, podrá usar instrumentos que provoquen formas diferenciadas de expresión del sujeto (González Rey, 2009c; González Rey et al., 2016). Esos instrumentos pueden representar una importante fuente para el desarrollo de hipótesis; así como para el desarrollo de momentos de conversación cargados de sentido subjetivo. Estos momentos pueden ser para nuevas producciones de sentido subjetivo en el curso de la psicoterapia. Con las hipótesis no se 
pretende acceder a una "verdad" del sujeto, sino facilitar la emergencia de producciones subjetivas alternativas a aquellas dominantes, asociadas al problema que demanda el espacio terapéutico (González Rey, 2009c; González Rey et al., 2016).

Desde esta mirada, se asume una postura crítica sobre el término patología en cuanto se refiere a la naturalización de un contenido como anormal (González Rey, 2015). La significación anterior lleva a definir lo patológico como un "objeto" separado del sujeto y del contexto histórico cultural de esa producción (González Rey, 2009c, 2015). Desde el discurso biomédico que ha atravesado a la psicología clínica se ha concebido la enfermedad como criterio explicativo y causal, en lugar de comprenderla como génesis de una posible forma de subjetividad (González Rey, 2015). Se busca en la patología una explicación del carácter inhumano del acto, y no la condición subjetiva de la persona que lo hace posible. Se quiere descartar al acto humano a través de su patologización (González Rey, 2009c, 2015).

En este sentido, en esta perspectiva se propone la elaboración de criterios que permitan diferenciar una psique saludable, de otra generadora de daños (González Rey, 2009c). La salud psíquica se define por la posibilidad de generar nuevos sentidos subjetivos y configuraciones subjetivas que den paso al desarrollo subjetivo en el transcurso de los conflictos que el sujeto enfrenta (González Rey, 2011). Según este autor, la experiencia humana es conflictiva no por su carácter en sí, sino por las propias producciones subjetivas generadas por el sujeto en el curso de su experiencia. Sin embargo, el límite entre la salud y la patología estaría, en la imposibilidad del sujeto de producir nuevos sentidos subjetivos ante una condición que le afecta. Es decir, el trastorno se considera un tipo de configuración subjetiva dominante que produce sentidos subjetivos que obstaculizan e impiden la capacidad del individuo de generar nuevos sentidos subjetivos ante nuevas experiencias (González Rey, 2015; González Rey \& Mitjans Martínez, 2017). Este planteamiento centra el enfoque en la singularidad, rechazando la idea de que los trastornos están definidos por emociones específicas y de contenido universal (González Rey, 2015).

Los 'trastornos' no son una entidad, sino que representan sistemas recursivos de sentidos subjetivos, que en su desarrollo se rigen por un momento dominante de la configuración que los integran (González Rey, 2009c, 2011). Esta organización patogénica se alimenta de las formas de vida actuales del sujeto y de sus propias representaciones y posiciones sobre lo que está viviendo (González Rey, 2009c, 2011). De esta forma, el individuo naturaliza sus formas de vida y sus representaciones, 
convirtiéndose en una fuente de perpetuación de su propio malestar (González Rey, 2009c, 2011). La psicoterapia representa entonces (entre otras) un camino orientado a “descentrar” el individuo de sus prácticas dominantes actuales (González Rey, 2009c).

Según planteó González Rey (2015), esto apunta a la necesidad de ir más allá de la categorización de conductas como patologías para acentuar la comprensión de las configuraciones subjetivas de las enfermedades. Esto, con el fin de identificar configuraciones subjetivas sanas que permitan la acción activa de la persona en el control de las enfermedades crónicas y en el desarrollo de recursos que les permitan una vida activa, alejándola de la condición de paciente (González Rey, 2015).

Otra categoría que tiene implicaciones interesantes en el proceso de psicoterapia es la categoría de la subjetividad social y la forma en que lo sociocultural es una parte inseparable de los conceptos de configuración subjetiva y sentido subjetivo. Asumir la perspectiva expuesta en la teoría de subjetividad destaca la necesidad de no limitar las acciones profesionales del terapeuta al consultorio; es necesario actuar sobre la condición social de las personas, su modo de vida y el contexto dentro el cual realizan sus actividades (González Rey et al., 2016). Esto tiene varias implicaciones. En primer lugar, es necesario comprender la trama relacional dentro de la cual se genera el comportamiento que está siendo etiquetado como problemático o patológico (González Rey, 2015). Esto se debe a que son esas relaciones del sujeto donde se están generando los sentidos subjetivos vinculados a la configuración subjetiva en cuestión. De esta forma, antes de comenzar cualquier acción de cambio con la persona involucrada, es necesario comprender las dinámicas sociales vinculadas a la configuración subjetiva de la “enfermedad” (González Rey, 2015).

En segundo lugar, asuntos como el uso del tiempo, las actividades cotidianas que caracterizan su modo de vida, la calidad de sus sistemas de relación y el desarrollo de nuevos procesos de socialización y realización personal deben formar parte del foco de atención del proceso de terapia (González Rey, 2015; González Rey et al., 2016). Finalmente, el concepto de subjetividad social implica la posibilidad de analizar el funcionamiento de las instituciones para intervenir sobre estas. González Rey (2009c, 2015) señaló que la psicoterapia siempre está implicada en un espacio de subjetividad social, ella se realiza dentro de discursos que expresan sentidos subjetivos y procesos simbólicos que están más allá del propio espacio terapéutico. No obstante, debido a que la subjetividad social también se articula en diversos espacios sociales fuera del espacio del consultorio, la psicoterapia entonces puede ser realizada fuera del mismo (González 
Rey et al., 2016). La propuesta de este autor es que los espacios sociales tales como la sala de clases, el hospital, los tribunales, las instituciones, los ambientes laborales, la comunidad, los grupos sociales, la familia, entre otros, pueden ser considerados escenarios sociales terapéuticos.

\section{Propuesta investigativa: La Epistemología Cualitativa y sus implicaciones para la investigación en la psicoterapia}

Un último aspecto en torno a las implicaciones de esta teoría para la práctica clínica es respecto a la investigación. Entre las aportaciones más significativas e interesantes que realizó González Rey (2000b, 2007a), se encuentra la elaboración de la Epistemología Cualitativa, para dar cuenta de la naturaleza cualitativamente diferente de la subjetividad como objeto de estudio. Esta epistemología ha nutrido parte de su concepción sobre la aplicación de su teoría a la psicoterapia. En su abordaje teórico propuso tres principios centrales: (1) El carácter constructivo interpretativo del conocimiento; (2) la legitimación de lo singular como instancia de producción del conocimiento científico; y (3) la comprensión de la investigación en las ciencias sociales como un proceso de comunicación (González Rey, 2007a; González Rey \& Mitjans Martínez, 2015; González Rey \& Patiño, 2017).

El carácter constructivo-interpretativo del conocimiento implica que este no es una aprehensión lineal de una realidad organizada según categorías universales, sino que es una producción humana (González Rey, 2007a). Específicamente, confiere valor al conocimiento, no por su correspondencia lineal e inmediata con lo "real", sino por la potencia de generar campos de inteligibilidad que permiten nuevas zonas de acción sobre la realidad. De igual manera, estos campos producen nuevas formas de acercarnos a la realidad mediante nuestras representaciones teóricas (González Rey, 2007a).

Este principio establece la necesidad de considerar la investigación como producción teórica (González Rey, 2007a). Esto no se limita a las teorías que son formas de conocimiento preexistentes en relación con el proceso de investigación. Más bien, debe ser entendido como la construcción de modelos en el proceso investigativo que generan inteligibilidad sobre lo estudiado. Aunque la teoría provee unos conceptos, estos son dinámicos y no tienen un contenido a priori. El contenido específico se construye a través de los modelos teóricos que el investigador elabora a lo largo de su investigación. Además, lo teórico tiene como eje central la actividad pensante y constructiva del investigador (González Rey, 2007a). 
El segundo principio, la legitimación de lo singular como instancia de producción del conocimiento científico, está estrechamente relacionada con una nueva comprensión de lo teórico (González Rey, 2007a). Esto se plantea en el sentido de que la legitimación de la información proveniente del caso singular transcurre mediante el modelo teórico que el investigador desarrolla en el proceso de investigación (González Rey, 2007a). La singularidad tiene un gran valor, ya que una de las características de la subjetividad es la marcada diferenciación de los individuos y los diferentes espacios sociales (González Rey, 2007a).

Por último, la investigación desde esta perspectiva se desarrolla como un proceso de comunicación (González Rey, 2007a). La comunicación es el espacio privilegiado en que el sujeto se inspira en sus diferentes formas de expresión simbólica (González Rey, 2007a). Cada una de sus formas diferenciadas de expresión son vías para el estudio de su subjetividad, de sus sentidos subjetivos, y de la manera en que sus condiciones sociales objetivas aparecen constituidas en este nivel. La comunicación será el camino por el cual los participantes de una investigación se convertirán en sujetos de esta, implicándose en el problema investigado a partir de sus intereses, deseos y contradicciones (González Rey, 2007a). A través de la expresión del sujeto, por medios diversos en donde esté implicado emocionalmente, el investigador podrá generar hipótesis utilizando las expresiones del participante como indicadores empíricos. Sobre estos indicadores construirá los sentidos subjetivos y las configuraciones subjetivas. Es decir, los sentidos subjetivos no son accesibles de forma directa en la narrativa del participante. Están ligados al proceso constructivo-interpretativo del investigador.

De esta forma, a través del caso singular y del proceso de comunicación, el investigador construye un modelo teórico que no está definido de antemano (González Rey, 2013b). Reiteramos que el concepto de configuración subjetiva no representa una entidad a priori para atribuir significados a la información que emerge en el curso de la investigación. La configuración subjetiva se construye en el curso de la investigación, representando un modelo teórico que genera inteligibilidad en el propio proceso de su construcción. Esta característica le otorga valor metodológico a la teoría, pues el modelo teórico va a representar el proceso de tránsito entre las construcciones hipotéticas y las conclusiones de la investigación, las que se definirán por el carácter final que tome el modelo teórico en dicho proceso (González Rey, 2013b).

Toda esta elaboración sobre la Epistemología Cualitativa resulta pertinente si consideramos que la academia ha tendido a destacar la investigación experimental, desde 
la epistemología positivista, en la psicología clínica (Kazdin, 1995). El método experimental es considerado el método predilecto ya que está caracterizado "por el control riguroso de variables que puedan contaminar la pureza de los procedimientos utilizados para conocer la realidad" (Lucca y Berrios, 2003, p.21). Sin restarle méritos a este enfoque convencional en el desarrollo de la psicología clínica, este acercamiento es incongruente con la propuesta de González Rey (2000b, 2007a).

El acercamiento que propuso González Rey busca comprender la problemática y trastornos psicológicos en la especificidad particular en cada sujeto (González Rey, 2009c, 2011b, 2015; Goulart \& González Rey, 2020). Las configuraciones subjetivas deben ser construidas teóricamente por el investigador en cada caso particular y esto solo es posible a través de un proceso de comunicación con el paciente. Todo esto hace evidente que el sujeto particular tiene un lugar central en esta mirada a diferencia de la epistemología positivista, en la cual el lugar central lo tiene el sujeto abstracto y promedio de las estadísticas (De Oliveira et al., 2017).

La teoría de la subjetividad con la Epistemología Cualitativa presenta el espacio de psicoterapia como uno privilegiado para la investigación. Según los principios expuestos, el caso singular adquiere un valor indiscutible. Esto permitirá una relación recursiva entre la investigación y práctica. Cada sujeto que es atendido como parte de la psicoterapia constituirá un momento esencial para la investigación y para el desarrollo de la teoría, que a su vez redundará en nuevas transformaciones en la práctica. De esta forma, como señala Echemendia-Tocabens (2013), la psicología clínica y la psicoterapia se constituirían en espacios no solo de solución de problemas, sino de producción científica.

\section{Cuestionamientos y asuntos en agenda de la teoría de la subjetividad}

Una vez presentada la teoría de la subjetividad y la Epistemología Cualitativa con sus implicaciones para la psicoterapia, identificamos algunas interrogantes. En primer lugar, es pertinente discutir el asunto de la PHC como una escuela de psicoterapia. González Rey (2009c) y autores como Echemendía-Tocabens (2013) y Zaldívar Pérez (1999) se opusieron a la idea del desarrollo de una escuela histórico cultural en la psicoterapia. Aunque comprendamos la resistencia de estos autores debido al dogmatismo o cierre teórico que esto pudiese crear, es necesario desarrollar las articulaciones académicas para generar un núcleo común para una discusión sistemática de la psicoterapia y la PHC. El que eventualmente se constituya en un acercamiento propio (o escuela de pensamiento) no tiene por qué ser un problema. Las dificultades de 
dogmatización pueden ocurrir aun si se implementa el marco de la PHC como teoría integradora de otras teorías como propone Echemendía-Tocabens (2013). El asunto medular está en promover esa actitud crítica y de apertura que ha caracterizado a la PHC desde sus inicios y que han continuado en los autores contemporáneos. Dicha actitud es la de asumir de forma crítica la PHC y dialogar de forma abierta con otras teorías. Eso es lo que salvaguardará este acercamiento del dogmatismo y sectarismo, que ha caracterizado la búsqueda de hegemonía por parte de otras teorías psicológicas.

En segundo lugar, aunque la propuesta de la teoría de la subjetividad puede implementarse en el contexto clínico, es necesario el desarrollo y articulación de nuevas categorías más allá de las que González Rey (2002, 2009c) desarrolló. Partiendo de la teoría de la subjetividad, y de la PHC en general, se deben reconceptualizar y articular categorías, como por ejemplo la motivación y la necesidad. Aunque en cierta manera González Rey (1999, 2000b, 2002, 2014b, 2017) realizó esta reconceptualización, estas no se están integrando lo suficiente a su discurso teórico actual. A estas categorías se suman otras, como la zona de desarrollo próximo, la cual autores como EchemendíaTocabens (2013), Portes (2011) y Zaldívar Perez (1999) señalan su utilidad en el proceso terapéutico.

En tercer lugar, preguntamos, ¿Qué implicaciones puede tener en la aproximación de González Rey, la propuesta de Vygotsky, desarrollada en el segundo periodo de su obra, enfocada en las funciones psíquicas superiores y en la mediación semiótica? Parecería que la teoría de González Rey, al inspirarse en el primer y tercer momento de la obra de Vygotsky, es incompatible (aunque no completamente), con los desarrollos de Vygotsky más conocidos en Occidente. Esta interrogante se plantea ya que el mismo González Rey (2009a), y otros como Elhammoumi (2010) y Rodríguez Arocho (2010), apuestan a la representación sistémica de la teoría vygotskiana. Las elaboraciones que se han realizado inspiradas en el segundo momento de Vygotsky, aunque tienen un fuerte énfasis en lo cognitivo, no han dejado de ser un momento significativo del desarrollo en esta teoría en la actualidad. Quizás una integración de las dos psicologías de Vygotsky será necesario en un futuro (Álvarez \& del Río, 2007).

Finalmente, en términos metodológicos, siguiendo la Epistemología Cualitativa, González Rey (2007a) favoreció el estudio de caso como diseño investigativo para la investigación de la subjetividad. Esto lo hace ideal en el contexto clínico. No obstante, ¿Qué otros métodos más allá del estudio de caso pueden aportar este acercamiento a la clínica? ¿Cómo puede dialogar este acercamiento con la mirada positivista que ha 
prevalecido en la psicología clínica? Después de todo, las investigaciones y aportaciones desde esa mirada convencional han hecho sus aportes respectivos a la clínica.

\section{Conclusión}

Históricamente la PHC tuvo poca inserción en la psicología clínica y en la psicoterapia. Esto se debió a varias razones: 1) La traducción y difusión de la obra de Vygotsky a Occidente se dio en el contexto de la revolución cognitiva, lo cual dio paso (por diversas razones) a un sesgo cognitivista en la interpretación de su legado; 2) la ausencia de un interés directo de la PHC en la psicoterapia; 3) el hecho de que las disciplinas de 'ayuda' no estaban establecidas como prácticas culturales; 4) la falta de un desarrollo profundo de ciertos aspectos de la teoría de Vygotsky y 5) la ausencia de un desarrollo significativo de la psicología clínica y la psicopatología en la psicología rusa.

La elaboración en décadas recientes de la teoría de la subjetividad, desde la PHC, por González Rey (2002) representó un hito de desarrollo. Esta teoría muestra un gran potencial acerca de su posible aplicación al contexto clínico, como ya múltiples autores han explorado profundamente (De Oliveira et al., 2017; Díaz-Gómez et al., 2017; González Rey, 2009c, 2011b, 2015; González Rey et al., 2016; Goulart \& González Rey (2020). A esto se le suma la Epistemología Cualitativa que desarrolló González Rey, que permite brindarle un fundamento investigativo a la teoría de la subjetividad. Esto último, representa la posibilidad de considerar el espacio psicoterapéutico como espacio privilegiado de investigación, para mantener la teoría y la práctica en un continuo desarrollo.

Este es el comienzo de un largo camino por recorrer. Hay aún varios aspectos que deben pensarse y elaborarse para viabilizar la extensión de la PHC al campo de la psicología clínica. Ejemplo de esto es la necesidad de elaborar y emplear con precisión categorías como motivación, necesidad, entre otras categorías clásicas de la psicología repensadas desde esta propuesta teórica.

Al igual que otros autores, Vygotsky realizó importantes aportes a la psicología en general, los cuales pueden beneficiar al campo de la psicología clínica y la psicoterapia (Zaldívar Pérez, 1999). Esto demandará una labor creativa, crítica, sistemática y rigurosa por parte de la comunidad científica de la PHC, para elaborar propuestas teóricas diferenciadas. Estas propuestas deben ser consistentes con los principios elaborados por Vygotsky, reconociendo que categorías relevantes para la clínica no fueron desarrollarlas plenamente por él. Es pertinente retomar lo que este autor alcanzó a plantear en forma 
embrionaria, para desarrollarlo como un movimiento fértil que representa la PHC en la actualidad. Las propuestas de González Rey son punta de lanza para esa tarea. Esto no implica seguir la propuesta teórica de González Rey como final. Todo lo contrario, como bien lo planteó este teórico, sus abordajes representan solo una de muchas posibles elaboraciones a partir de los conceptos de Vygotsky. Lo medular en este aspecto es abrir a una discusión sistemática y crítica de la aplicación de la PHC a los campos de la psicología clínica y la psicoterapia. 


\section{Referencias}

Ageyev, V.S. (2003). Vygotsky in the mirror of cultural interpretations. En Kozulin, A., Gindis, B., Ageyev, V.S. \& Miller, S.M. (Eds.), Vygotski's educational theory in cultural context (pp. 432-449). Cambridge, MA: Cambridge University Press.

Alonso, A. (1998). Algunas aplicaciones de la teoría de L.S. Vigotski en psicologia clínica. Revista Cubana de Psicología, 15(2), 124-128.

Álvarez, A. \& del Río, P. (2007). Una introducción a las dos psicologías de Vygotsky. En P. del Río y a. Álvarez (Eds), Escritos sobre arte y educación creativa de L. S. Vygotsky (pp. 7- 20). Madrid: Fundación Infancia y Aprendizaje.

Daniels, H. (2001) Vygotsky and pedagogy. New York: RoutledgeFalmer.

De Oliveira, A., Goulart, D \& González Rey, L. (2017). Processos subjetivos da depressão: construindo caminhos alternativos em uma aproximação culturalhistórica. Fractal: Revista de Psicología, 29(3), 252-261. https://doi.org/10.22409/1984- 0292/v29i3/1411

Díaz-Gómez, A. \& González Rey, F. (2005). Subjetividad: una perspectiva histórico cultural. Conversación con el psicólogo cubano. Universitas Psychologica, 3, 373-383.

Díaz-Gómez, A., González-Rey, F., \& Arias-Cardona, A. (2017). Pensar el método en los procesos de investigación en subjetividad. Revista CES Psicología, 10(1), 129-145. http://dx.doi.org/10.21615/cesp.10.1.8

Echemendía-Tocabens, B. (2013). Psicoterapia y enfoque histórico-cultural. Aportes y desafíos. Santiago, 133, 85-98.

Elhammoumi, M. (2010). Is 'back to Vygotsky enough? The legacy of sociohistoricocultural psychology. Psicologia em Estudio 15(4), 661-673. http://dx.doi.org/10.1590/S1413-73722010000400002

Freud, S. (1978). Conferencias de introducción al psicoanálisis, conferencia 17: El sentido de los síntomas. En Obras completas, XVI (p. 328-342). Buenos Aires: Amorrortu Editores.

Gardner, H. (1988). La nueva ciencia de la mente: Historia de la revolución cognitiva. Buenos Aires: Editorial Paidós.

González Rey, F. (1999). La afectividad desde una perspectiva de la subjetividad. Psicología: Teoría e Pesquisa, 15(2), 127-134. http://dx.doi.org/10.1590/S0102-37721999000200005

González Rey, F. (2000a). El lugar de las emociones en la constitución social de lo psíquico: El aporte de Vygotsky. Educacao \& Sociedade, 70, 132-14. http://dx.doi.org/10.1590/S0101-73302000000200006

González Rey, F. (2000b). Investigación cualitativa en psicología: Rumbos y desafíos. México: Internacional Thomson Editores.

González Rey, F. (2002). Sujeto y subjetividad: Una aproximación históricocultural. México: Thomson Editores.

González Rey, F. (2007a). Investigación cualitativa y subjetividad. India: McGraw Hill.

González Rey, F. (2007b). Social and individual subjectivity from an historical cultural standpoint. Outlines. Critical Practice Studies, 9(2), 3-14. Recuperado de: https://tidsskrift.dk/outlines/article/view/1985

González Rey, F. (2008). Subjetividad social, sujeto y representaciones sociales. Revista Diversitas: Perspectivas en Psicología, 2(4), 225-243. 
González Rey, F. (2009a). Epistemología y ontología: Un debate necesario en la psicología hoy. Perspectiva Psicológica, 5(2), 205-224.

González Rey, F. (2009b). La significación de Vygotsky para la consideración de lo afectivo en la educación: las bases para la cuestión de la subjetividad. Revista Electrónica Actualidad Investigativas en Educación, 9, 1-25.

González Rey, F. (2009c). Psicoterapia, subjetividad y posmodernidad: Una aproximación desde Vygotsky hacia una perspectiva histórico-cultural. Buenos Aires, Argentina: Noveduc Libros.

González Rey, F. (2010a). El pensamiento de Vygotsky: repercusiones y consecuencias ausentes en una interpretación dominante. Novedades educativas, 230, 4-18.

González Rey, F. (2010b). Las categorías de sentido, sentido personal y sentido subjetivo en una perspectiva histórico-cultural: un camino hacia una nueva definición de subjetividad. Universitas Psychologica, 9(1), 241-253. Recuperado de: https://www.redalyc.org/articulo.oa?id=64712156019

González Rey, F. (2011a). Advancing on the Concept of Sense. In M. Hedegaard, A. Edwards \& M. Fleer (Eds.), Motives in children's development (pp.45-62). Cambridge: Cambridge University Press. http://dx.doi.org/10.1017/CBO9781139049474.005.

González Rey, F. (2011b). Sentidos subjetivos, lenguaje y sujeto: Avanzando en una perspectiva postracionalista en psicoterapia. Rivista di Psichiatria, 46, 310314. http://dx.doi.org/10.1708/1009.10978

González Rey, F (2013a). La subjetividad en una perspectiva histórico-cultural: avanzando un legado inconcluso. CS, 11(2), 642-676. http://dx.doi.org/10.1590/S0101-73302000000200006

González Rey, F. (2013b). Subjetividad, cultura e investigación cualitativa en psicología: la ciencia como producción culturalmente situada. Liminales, l(4), 13-36.

González Rey, F. (2013c). Subjetividad, cultura y psicología: Repasando un camino recorrido por la psicología en cuba. Alternativas Cubanas en Psicología, $1(1), 25-41$.

González Rey, F. (2014a). Advancing further the history of soviet psychology: Moving forward from dominant representations in western and soviet psychology. History of Psychology, 17(1), 60-78. https://doi.org/10.1037/a0035565

González Rey, F. (2014b). Human motivation in question: discussing emotions, motives, and subjectivity from a cultural-historical standpoint. Journal for the Theory of Social Behaviour, 45(4), 419-439. https://doi.org/10.1111/jtsb.12073

González Rey, F. (2015). A saúde na trama complexa da cultura, das instituições e da subjetividade. En F. González Rey \& J. Bizerril (Eds.), Saúde, cultura e subjetividade: Uma referência interdiciplinar (pp.9-34). Brasília: UniCEUB.

González Rey, F. (2016). El pensamiento de Vygotski: momentos, contradicciones y desarrollo. Summa Psicológica UST, 13(1), 7-18. https://doi.org/10.18774/summa-vol13.num1-278

González Rey, F. (2017). Subjectivity and discourse: Complementary topics for a critical psychology. Annual Review of Critical Psychology, 13, 1-12. Recuperado de https://thediscourseunit.files.wordpress.com/2017/08/arcpfernandog.pdf 
González Rey, F. \& Mitjans Martínez, A. (2015). Una epistemología para el estudio de la subjetividad: Sus implicaciones metodológicas. Psicoperspectivas, 15(1), 5-16. http://dx.doi.org/10.5027/psicoperspectivas-Vol15-Issue1fulltext-667

González Rey, F. y Mitjans Martínez, A. (2017). El desarrollo de la subjetividad: una alternativa frente a las teorías del desarrollo psíquico. Papeles de Trabajo sobre Cultura, Educación y Desarrollo Humano, 13(2), 3-20.

González Rey, F. \& Patiño, J. (2017). La Epistemología Cualitativa y el estudio de la subjetividad en una perspectiva cultural histórica: conversación con Fernando González Rey. Revista de Estudios Sociales, 60, 120-128. https://dx.doi.org/10.7440/res60.2017.10

González Rey, F., Goulart, D. \& Bezerra, M. (2016). Ação profissional e subjetividade: para além do conceito de intervenção profissional na psicologia. Educação, 39(número especial), 54-65.

González Rey, F., Mitjans Martínez, A. \& Bezerra, M. (2016). Psicología en la educación: implicaciones de la subjetividad en una perspectiva cultural histórica. Revista Puertorriqueña de Psicología, 27(2), 260-274.

Goulart, D. \& González Rey, F. (2020). Subjetividad, sujeto y salud mental: un estudio de caso para más allá de la lógica de la patología. Alternativas Cubanas en Psicología, 8(23), 14- 35.

Hernández, O. (2008). La subjetividad desde la perspectiva histórico-cultural: un tránsito desde el pensamiento dialéctico al pensamiento complejo. Revista Colombiana de Psicología, 17, 147-160.

Hernández, A. (2010). La vivencia como categoría de análisis para la evaluación y el diagnóstico del desarrollo psicológico. PsicoPediaHoy, 12(12). Recuperado en http://psicopediahoy.com/vivencia-como-categoria-de-analisis-psicologia/

Holzman, L. (2014). "Vygotskian-izing" psychotherapy. Mind, Culture, and Activity, 21(3), 184-199. https://doi.org/10.1080/10749039.2013.828312

Kats, N. \& Hadas, N. (1995). Cognitive rehabilitation: Occupational therapy models for intervention in psychiatry. Psychiatric Rehabilitation Journal, 19(2), 2936. http://dx.doi.org/10.1037/h0095443

Kazdin, A. (1995) Methods of psychotherapy research (Chapter 21). In B. Bongar \& L.E. Beutler (Eds.), Comprehensive textbook of psychotherapy: Theory and practice. New York: Oxford.

Kozulin, A. (1990). The concept of regression and Vygotskian Developmental theory. Developmental Review, 10, 218-238. https://doi.org/10.1016/02732297(90)90011-R

Lucca, N. \& Berríos, R. (2003). Investigación Cualitativa en Educación y Ciencias Sociales. Hato Rey, PR: Publicaciones Puertorriqueñas, Inc.

Miller, G. (2003). The cognitive Revolution: A historical perspective. Trends in Cognitive Science, 7(3), 141-144. http://dx.doi.org/10.1016/S13646613(03)00029-9

Miltenburg, R. \& Singer, E. (1999). Culturally mediated learning and the development of self regulation by survivors of child abuse: A Vygotskian approach to support of survivors of child abuse. Human Development, 42, 117. http://dx.doi.org/10.1159/000022604

Portes, P. (2011). Vygotsky's significance in advancing counseling and psychotherapy. En P. Portes \& S. Salas (Eds.) Vygotsky in $21^{\text {st }}$ century society: Advances in cultural historical theory and praxis with non-dominant communities (pp.113-130). New York: Peter Lang Publishing. 
Rivière, A. (1987). El sujeto de la psicología cognitiva. Alianza Editorial

Rodríguez Arocho, W. (1994). Trasfondo histórico y filosófico del estudio de la cognición. Crecemos, 1, 6-9.

Rodríguez Arocho, W. (1995). Jean y L. S. Vygotski: Pertinencia de sus contribuciones a la psicología contemporánea. Revista Puertorriqueña de Psicología, 10(1), 1-18.

Rodríguez Arocho, W. (1999). El legado de Vygotski de Piaget a la educación. Revista Latinoamericana de Psicologia, 31(3), 477-489.

Rodríguez-Arocho, W. (2006). El constructivismo: Una invitación al análisis de sus antecedentes, vertientes y críticas.Pedagogía, 39(1), 12-28.

Rodríguez Arocho, W. (2010). Los conceptos de vivencia y situación social del desarrollo: reflexiones en torno a su lugar en el modelo teórico de Lev S. Vygotski. En S. Aburto \& C. Meza (Eds.), Tutoría para el desarrollo humano: Enfoques (pp.73-92). México: Universidad Autónoma de Nuevo León.

Rodríguez Arocho, W. (2013). El lugar de la afectividad en la psicologia de Vygotski: Reflexividad histórico y reivindicación. Propósitos y Representaciones, 1(2), 105-129.

Ryle, A. (1991). Object relations theory and activity theory: A proposed link by the way of procedural sequence model. British Journal of Medical Psychology, 64, 307-316.

Van der Veer, R. \& Valsiner, J. (1991). Understanding Vygotsky: A quest for synthesis. Cambridge, MA: Blackwell.

Varela, F. (1988). Conocer: Las ciencias cognitivas. Tendencias y perspectivas. Madrid: Gedisa Editorial.

Vygotsky, L. S. (1982/1993). Obras escogidas, Vol. II, Problemas de la psicología general. Madrid: Aprendizaje-Visor.

Vygotsky, L. S. (1934). The problem of the environment. Recuperado de http://www.marxists.org/archive/vygotsky/works/1934/environment.htm

Wilson, A. \& Weinstein, L. (1992). An investigation into some implications of a Vygotskian perspective on the origins of mind: Psychoanalysis and the Vygotskian psychology, Part 1. Journal of the American Psychoanalytic Association, 40, 349-370. https://doi.org/10.1177/000306519204000203

Zaldívar Pérez, D. F. (1999). Psicoterapia y orientación histórico cultural. Revista Cubana de Psicología, 16(3), 7-17. 